\title{
103. Indikationen zur Relaparotomie beim Morbus Crohn
}

\author{
H. Krieg, H. Brünner, M. Neher und G. Gamstätter \\ Chirurg. Univ.-Klinik, Langenbeckstr. 1, D-6500 Mainz \\ Indications for Relaparotomy in Crohn's Disease
}

Summary. Relaparotomy is needed in various clinical courses and complications of Crohn's disease. In a series of 153 of the authors' patients with Crohn's disease a total of 310 operations were performed, 157 of them being relaparotomies. If a more conservative treatment proves unsuccessful, relaparotomy is indicated in all cases of chronic Crohn's disease with severe complications and other general postoperative complications not primarily related to Crohn's disease.

Key words: Disease, Crohn's - Indications - Relaparotomy.

Zusammenfassung. Unterschiedliche klinische Verlaufsformen des Morbus Crohn mit zahlreichen Komplikationen zwingen den Chirurgen zur Relaparotomie. Wir berichten über 153 Morbus Crohn-Patienten, bei denen insgesamt 310 Operationen, darunter 157 Relaparotomien durchgeführt wurden. Die Indikation zur Reintervention ist bei allen chronisch-komplizierten Verläufen und nach postoperativen Crohn-unspezifischen Komplikationen gegeben, wenn die konservative Therapie erfolglos war.

Schlïsselwörter: Morbus Crohn - Indikationen - Relaparotomie.

\section{Postoperative Komplikationen nach Notfallresektion bei Colitis ulcerosa}

\author{
W. Stock und W. G. Kramme \\ Chirurgische Universitätsklinik, Köln-Lindenthal
}

\section{Postoperative Complications after Emergency Resection in Ulcerative Colitis}

Summary. Of 44 patients with ulcerative colitis, 17 had to undergo resection under emergency conditions because of toxic dilatation and fulminant bleeding of the colon. Eight patients died within 10 days due to the operative disruption with fecal soiling resulting in fatal sepsis. These severe complications can be avoided by the method of Turnbull, i. e. Ioop ileostomy and transversum and sigmoid decompression colostomy in the toxic dilatation phase. Proctocolectomy can be performed without risk 6 months later.

Key words: Colitis, ulcerative - Dilatation, toxic - Peritonitis, fecal - Operation, Turnbull's.

Zusammenfassung. Wegen toxischer Dilatation und massiver Blutung des Colons bei Colitis ulcerosa mußte bei 17 von 44 Patienten unter Notfallbedingungen operiert werden. 8 Patienten verstarben innerhalb von durchschnittlich 10 Tagen an den Folgen der kotigen Peritonitis nach Colonperforation. Zur Verhütung dieser schweren Komplikationen gilt die Anlage einer doppelläufigen Ileostomie sowie einer Transversum- und Sigmadekompressionskolostomie nach Turnbull mit zweizeitiger Proktokolektomie nach 6 Monaten als Methode der Wahl.

Schlïsselwörter: Colitis ulcerosa - Toxische Dilatation - Kotige Peritonitis - Turnbullsche Operation. 